\title{
An Additively Manufactured 3D Antenna-in- Package with Quasi-Isotropic Radiation for Marine Animals Monitoring System
}

\author{
Hanguang Liao, Student Member, IEEE, Qingle Zhang, Muhammad Akram Karimi, Yen-Hung Kuo, \\ Nidhi Mishra and Atif Shamim, Senior Member, IEEE
}

\begin{abstract}
A low-cost and additively manufactured 3D Antenna-in-Package (AiP) with quasi-isotropic radiation is proposed for a marine animals monitoring system. The antenna is based on a meandered dipole folded as a split ring resonator (SRR) structure, which can generate simultaneously a pair of orthogonal electric and magnetic dipoles, thus providing a quasi-isotropic radiation pattern. The antenna (integrated with a balun) has been inkjet-printed on a 3D-printed buoyant cone structure, which acts also as the system package to house the electronics and the battery. The antenna designed at $2.4 \mathrm{GHz}$ is electrically small, with a $\boldsymbol{k a}=$ 0.49 , and has a bandwidth of $70 \mathrm{MHz}(2.9 \%)$. The measured gain deviation of the antenna (maximum to minimum) is near $3 \mathrm{~dB}$ in bandwidth, thus qualifying it as a quasi-isotropic antenna. Field tests of the antenna in the active state (integrated with the electronics) confirm a reliable communication range of $240 \mathrm{~m}$ in any direction in the azimuthal plane.
\end{abstract}

Index Terms-additively manufactured, quasi-isotropic radiation, electrically small, split ring resonator (SRR), antennain-package (AiP).

\section{INTRODUCTION}

W ITH shifting climatic conditions and enhanced human activities, sea life is being affected. To protect and preserve the ocean environment and marine animals, efforts are being made to study these changes by sensing environmental parameters (such as water density, temperature, pressure, oxygen level and pollutants) in addition to the activities of various marine animals [1]. However, data acquisition from such sensors in the marine environment has always been difficult. Typically, expensive, complex and bulky methods such as hundreds of kilometers of optical fiber and advanced pop-up buoys are employed for this purpose [2]. A prospective solution is depicted in Fig. 1, in which hundreds of receivers float in the intended study area (typically a marine-protected area) and can collect data from the wireless sensor tags on marine animals every time they appear on the surface. Afterward, the data from the floating receivers can be either offloaded to a drone or sent via a GSM connection, if available.

This publication is based upon work supported by the King Abdullah University of Science and Technology (KAUST) Office of Sponsored Research (OSR) under Award No. Sensor Innovation Initiative OSR-2015-Sensors-2707.

Hanguang Liao, QingLe Zhang, Yen-Hung Kuo, Nidhi Mishra and Atif Shamim are with the Department of Electrical Engineering, King Abdullah

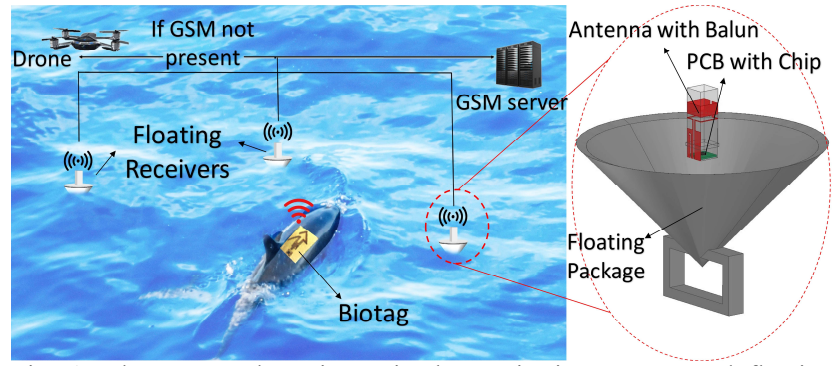

Fig. 1. The proposed marine animals monitoring system and floating receiver view.

The key requirements for such floating receivers are their buoyancy, low cost and equally successful communication in all directions. Thus, a quasi-isotropic antenna is desirable.

For lower cost and compactness, the AiP concept can be beneficial [3]. In this concept, an antenna is realized on the package of the electronics, making the package functional and part of the system rather than a mere cover for the sensitive electronics. As a result, considerable cost and space savings can be achieved. Additional cost savings can be attained by adopting additive manufacturing, which avoids the use of expensive masks and material wastage [4]. Though not for marine monitoring applications, various 3D quasi-isotropic antennas have been proposed [5-10]. These are summarized in Table I. It can be seen that few works utilize the AiP concept and additive manufacturing. Some of the reported designs [56] feature large electrical sizes $(k a>1)$ and still do not provide adequate isotropicity (gain deviation $>4$ ). Other designs [7-9], though electrically small, still do not possess an isotropicity up to the mark. Most of the works do not demonstrate integration of electronics and isotropicity in active testing. It is well known that embedded electronics and battery can influence the radiation pattern and isotropicity. Finally, except for [8], none of these designs are suitable for marine monitoring application, as they do not fulfill the buoyancy requirements. However, even in [8], the antenna performance is affected by water due to nonoptimized floating geometry.

In this letter, we propose a compact, buoyant, additively manufactured 3D AiP with a decent quasi-isotropic radiation

University of Science and Technology(KAUST), Thuwal 23955-6900, Saudi Arabia (e-mail: hanguang.liao@kaust.edu.sa, atif.shamim@kaust.edu.sa). 
TABLE I

COMPARISON OF TYPICAL QUASI-ISOTROPIC ANTENNAS

\begin{tabular}{|c|c|c|c|c|c|c|c|}
\hline $\begin{array}{c}\text { Referenc } \\
\text { e }\end{array}$ & Electrical Size $(k a)$ & $\begin{array}{l}\text { Measured Gain } \\
\text { Deviation }(\mathrm{dB})\end{array}$ & $\begin{array}{c}\text { Additive } \\
\text { Fabrication }\end{array}$ & AiP & $\begin{array}{c}\text { Testing with } \\
\text { Integrated } \\
\text { Electronics }\end{array}$ & Buoyancy & $\begin{array}{c}\text { Communication } \\
\text { Range }(\mathrm{m})\end{array}$ \\
\hline$[5]$ & 1.55 & NA & YES & YES & $\mathrm{NO}$ & $\mathrm{NO}$ & NA \\
\hline [6] & 2.10 & 8.9 and 10 & YES & YES & $\mathrm{NO}$ & $\mathrm{NO}$ & NA \\
\hline [7] & 0.41 & 5.2 & $\mathrm{NO}$ & $\mathrm{NO}$ & $\mathrm{NO}$ & $\mathrm{NO}$ & NA \\
\hline [8] & 0.54 & $<7$ & YES & YES & YES & YES & 45 \\
\hline [9] & 0.47 & 4.4 and 5.7 & $\mathrm{NO}$ & $\mathrm{NO}$ & $\mathrm{NO}$ & $\mathrm{NO}$ & NA \\
\hline$[10]$ & 0.98 & 1.9 & $\mathrm{NO}$ & $\mathrm{NO}$ & $\mathrm{NO}$ & $\mathrm{NO}$ & NA \\
\hline $\begin{array}{l}\text { This } \\
\text { Work }\end{array}$ & 0.49 & 3.3 & YES & YES & YES & YES & 240 \\
\hline
\end{tabular}

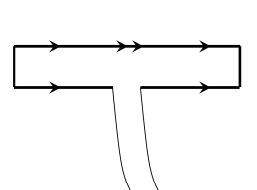

(a)

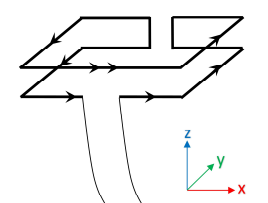

(b)

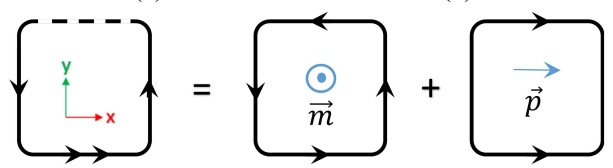

(c)

Fig. 2. Current distribution: (a) folded dipole antenna; (b) meandered folded dipole antenna; (c) analysis model of SRR current.

pattern. The buoyancy and low-cost requirements have been fulfilled by a custom floating structure that has been additively manufactured. The antenna has been realized on the top portion of the floating structure, which remains always outside the water. For the quasi-isotropic radiation pattern and electrically small size, a split ring resonator (SRR) approach has been utilized in the antenna design. Resultingly, a pair of orthogonal electric dipole and equivalent magnetic dipoles can be excited simultaneously, and a quasi-isotropic radiation pattern can be achieved. The 3D-printed cuboid and the floating structure is not only the substrate for the antenna but also houses the driving electronics and the battery, thus providing the necessary packaging function. A decent isotropicity is achieved (maximum gain deviation $<4$ in bandwidth) in measurements, and the buoyant receiver can communicate up to a range of 240 $\mathrm{m}$ in any direction in the azimuthal plane.

\section{QUASI-ISOTROPIC ANTENNA DESIGN}

\section{A. Design Strategy}

The design strategy is displayed in Fig. 2. A simple halfwavelength folded dipole, with its current distribution, is illustrated in Fig. 2(a). When this folded dipole is meandered in such a way that it can be wrapped on a 3D cuboid structure, it takes the form of a typical SRR, as presented in Fig. 2(b). The meandered folded dipole is based on the SRR structure to take its advantage, such as electrically small size [11-12]. The ratio of the upper and lower dipole widths can be selected to facilitate impedance matching [13]. As can be viewed in Fig. 2(a, b), the current distributions for the folded and meandered folded dipoles are similar (i.e., maximum current at the feed point and minimum current at the end of each arm). This SRR structure generates simultaneously a pair of orthogonal electrical $(\vec{p})$ and magnetic $(\vec{m})$ dipoles, as displayed in Fig. 2(c). The radiation patterns from the $\pm x$ axis electrical dipole and $\pm z$ axis

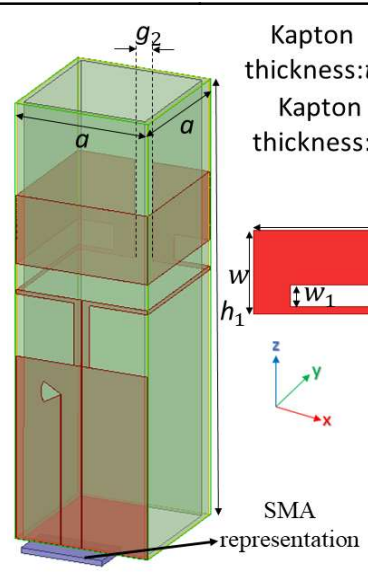

(a)
Fig. 3. Geometry of the AiP: (a) perspective view; (b) 4-layer stack-up; (c) planar view of antenna with balun; (d) floating structure view. $a=13, w=$ 9.5, $w_{c}=0.7, w_{1}=2.5, w_{2}=0.4, w_{3}=6.5, w_{4}=2, w_{5}=2.1, g_{1}=$ $0.1, g_{2}=1.8, h_{1}=4.2, h_{2}=17.6, h_{3}=14.6, r=2, t_{1}=0.03, t_{2}=$ $0.09, t_{s}=0.47, t_{a}=0.005, L_{c}=6, \mathrm{c}=\frac{2}{3} \pi, d_{c}=150, h_{4}=75, h_{5}=$ $36, h_{6}=23, W_{h}=11, L_{h}=62, t_{p}=6$ (unit: $\mathrm{mm}$ ).

magnetic dipole compensate each other's nulls, thus forming a quasi-isotropic radiation pattern.

\section{B. Antenna Design and Simulation Model}

For the SRR antenna implementation, a 3D printable cuboid structure is selected, as illustrated in Fig. 3(a). The 3D printable material is Vero Black Plus $\left(\varepsilon_{r}=2.8, \tan \delta=0.02\right)$, and the cuboid acts not only as part of the package but also as the substrate of the antenna, whose thickness $t_{s}=0.47 \mathrm{~mm}$ is chosen as a compromise to acceptable dielectric loss and decent robustness. Further, this thickness also facilitates $50 \Omega$ matching with realizable dimensions. The antenna stack-up, presented in Fig. 3(b), comprises three more layers, in addition to the base Vero material. These layers include a $30 \mu \mathrm{m}$ Kapton $\left(\varepsilon_{r}=3, \tan \delta=0.007\right.$ ) layer (for a low-loss material suitable for inkjet printing of the metallic antenna) and a metallic layer (for antenna geometry implementation), which is followed by a $90 \mu \mathrm{m}$ Kapton layer (to act as a waterproofing layer). This arrangement is intended to facilitate smooth realization of the antenna in addition to its protection in the water environment. The dimensions of the proposed antenna and package are displayed in Fig. 3. The conductor component of the antenna is designed to be fabricated by inkjet printing using nano-silver ink, which has a conductivity of $\sigma=5.0 \times 10^{6} \mathrm{~S} / \mathrm{m}$ and a thickness of $t_{a}=0.005 \mathrm{~mm}$ after curing. An integrated balun has also been designed for testing the differential antenna with single-ended equipment. Since a Sub-Miniature version A 


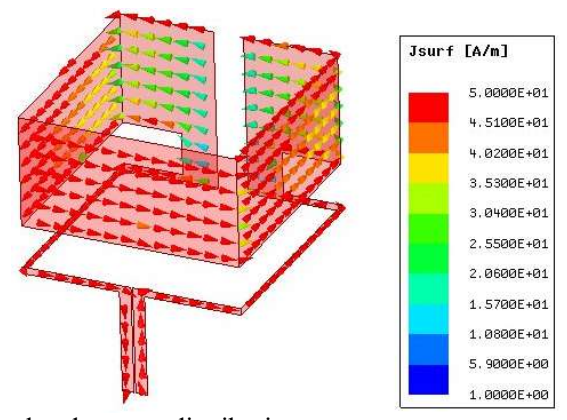

Fig. 4. Simulated current distribution.

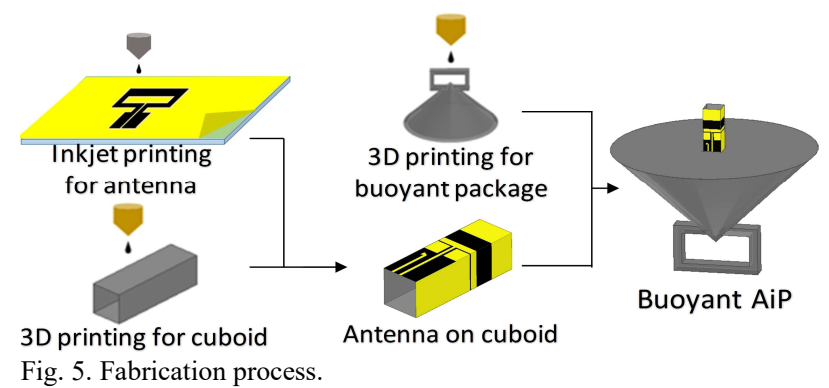

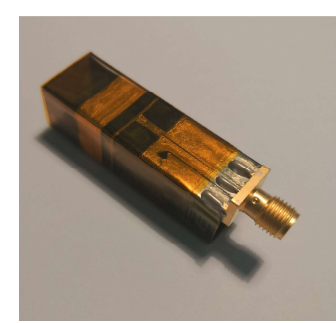

(a)

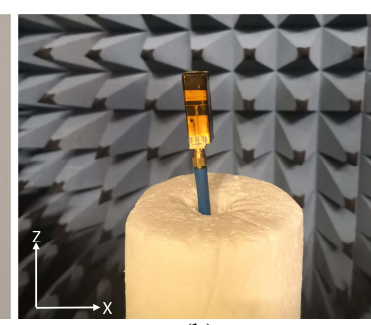

(b)
Fig. 6. Photos: (a) fabricated antenna on cuboid; (b) radiation pattern measurement in anechoic chamber.

(SMA) connector is primarily metallic, a perfect electric conductor (PEC) is employed at the bottom of the cuboid structure to replicate the SMA connector in simulations, as illustrated at the bottom of Fig. 3(a). The floating package (showed in Fig. 3 (d)) is a combination of a cone and a rectangular handle shaped structure. The cone provides both the buoyancy and the stability in water, while the handle adds weight to the package to further stabilize the whole system in water. The cone structure and the rectangular "handle" have the same thickness, $t_{p}=6 \mathrm{~mm}$, and the "handle" width is $W_{h}=$ $11 \mathrm{~mm}$. High-frequency structure simulator (HFSS) software is utilized to simulate the antenna. The simulated current distribution on the antenna surface is displayed in Fig. 4. As expected, the current distribution on the SRR structure is similar to the distribution presented in Fig. 2.

\section{ANTENNA FABRICATION AND MEASUREMENT}

\section{A. Fabrication Process}

The fabrication process is illustrated in Fig. 5. The floating package (comprising the bottom cone and the top cuboid structure) has been fabricated in two independent steps via the Objet 260 Connex 3D printer by Stratasys ${ }^{\circledR}$. The initial plan was to inkjet print the metallic part directly on the 3D-printed cuboid structure. However, due to the higher loss tangent of the Vero material and the high surface roughness, it was decided to change the metallic inkjet printing on a thin Kapton tape. The

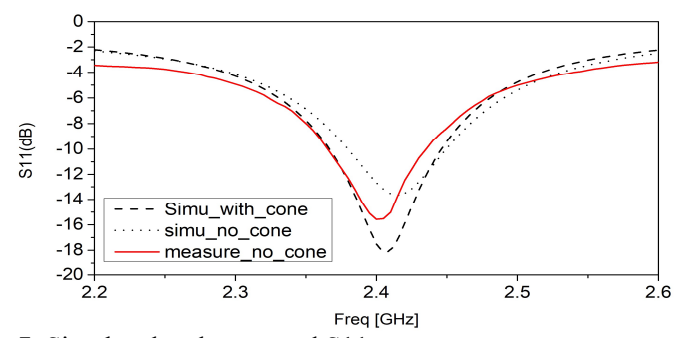

Fig. 7. Simulated and measured S11 parameter.

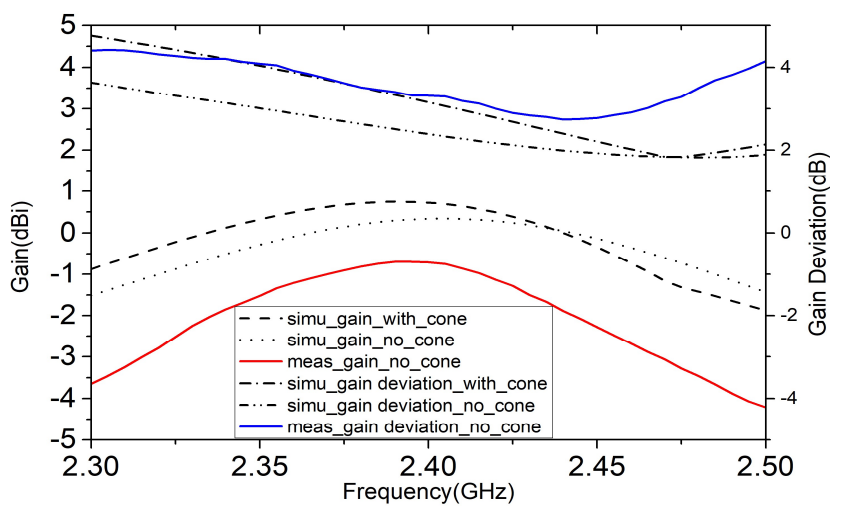

Fig. 8. Simulated and measured realized gain and gain deviation.

$30 \mu \mathrm{m}$ Kapton tape is attached temporarily to a glass slide for ease of inkjet printing. The conductor part is then printed with a Dimatix Materials inkjet printer (DMP-2831) using nanosilver ink, which is then cured at $150^{\circ} \mathrm{C}$ for one hour. The Kapton tape is then removed from the glass slide and is attached to the 3D-printed cuboid. Another $90 \mu \mathrm{m}$ Kapton tape is wrapped on the cuboid for waterproofing. The bottom cone portion is later integrated with the top cuboid portion. To test, an SMA connector is attached using Circuitworks ${ }^{\circledR}$ Conductive Epoxy. The prototype of the antenna with integrated balun is presented in Fig. 6(a).

\section{B. Passive Test Results}

The prototype is measured for its impedance properties via a KEYSIGHT ${ }^{\circledR}$ E8363C PNA Network Analyzer. The radiation properties measurements were conducted in a SATIMO ${ }^{\circledR}$ Star Lab Anechoic Chamber, as displayed in Fig. 6(b). The $S_{11}$ parameter of the proposed antenna is illustrated in Fig. 7. The measured resonance frequency of $2.405 \mathrm{GHz}$ is close to the simulated value of $2.415 \mathrm{GHz}$. Both the simulated and the measured results demonstrate a $10 \mathrm{~dB}$ impedance bandwidth of $70 \mathrm{MHz}(2.9 \%$ at $2.4 \mathrm{GHz})$. Fig. 8 depicts the realized gain in addition to the gain deviation. The maximum realized gains in the simulations and measurements are $0.34 \mathrm{dBi}$ and $-0.74 \mathrm{dBi}$, respectively. The measured gain is approximately $1 \mathrm{~dB}$ lower than the simulated value in the desired bandwidth. This can be attributed to the lower conductivity of the sensitive metallic ink, which heavily depends on the number of printed layers, curing temperature and duration, and some non-idealities in inkjet printing process such as coffee ring effect, etc. [14]. In measurements, gain deviation varies from $2.7 \mathrm{~dB}$ to $3.6 \mathrm{~dB}$ in bandwidth, which confirms adequate isotropic behavior from the antenna. To investigate the influence of the floating package, antenna simulations have been done with and without the cone structure (Fig. 7 and Fig. 8). As can be seen the 


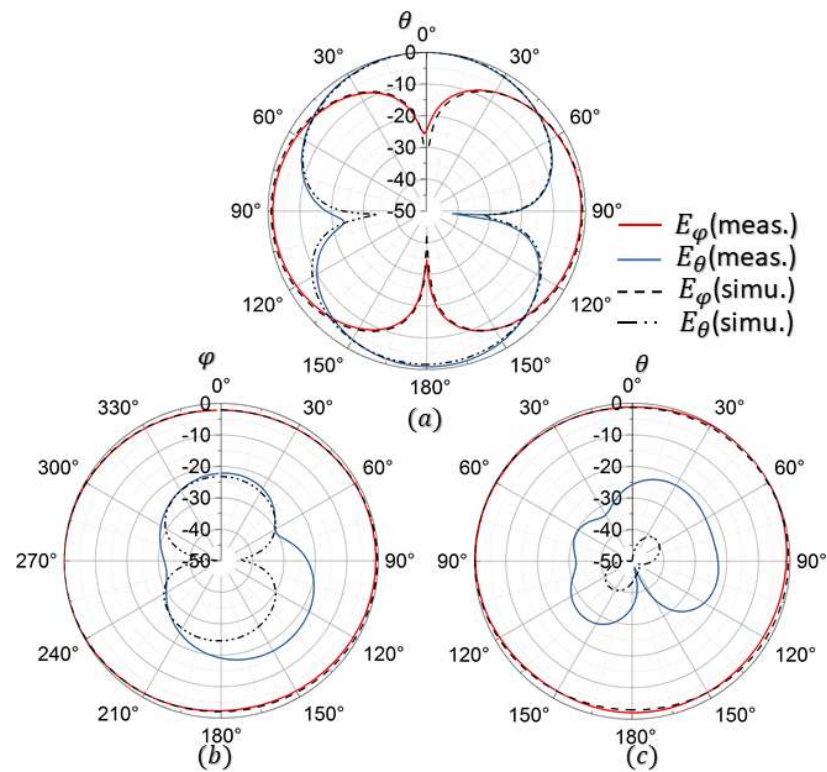

Fig. 9. Normalized simulated $(2.415 \mathrm{GHz})$ and measured $(2.405 \mathrm{GHz})$ radiation pattern: (a) $x z$-plane; (b) $x y$-plane; (c) $y z$-plane.

buoyant package does not affect the antenna performance much. The antenna is still well matched at the desired frequency $(2.4 \mathrm{GHz})$ with decent bandwidth, and the gain and gain deviation are also similar to the case with no package in the simulations.

The simulated and measured normalized radiation patterns in the elevation planes ( $x z$-plane and $y z$-plane) and the azimuthal plane ( $x y$-plane) are presented in Fig. 9. Consistent with the operation principle of the proposed antenna (illustrated in Fig. 2), Fig. 9(a) reveals that $E_{\theta}$ from the electric dipole and $E_{\varphi}$ from the magnetic dipole compensate each other's nulls, thus achieving a near omnidirectional radiation in the $x z$-plane. In Fig. 9(b, c), both near omnidirectional radiation patterns in $x y$-plane and $y z$-plane are formed by the $E_{\varphi}$ component from both electric and magnetic dipoles. The cross-polarization levels are approximately $15 \mathrm{~dB}$ lower than the co-polarization levels. All the radiation patterns, presented in Fig. 9, demonstrate an adequate correlation between simulations and measurements.

\section{Active Test Results}

The integrated receiver is illustrated in Fig. 10(a), in which the driving electronics and a $3.7 \mathrm{~V}$ Lithium-ion battery are placed inside the cone portion of the buoyant package. The driving circuit comprises a Bluetooth chip from Nordic Semiconductor ${ }^{\circledR}$ nRF52832, for which a custom PCB has been designed. To evaluate the system with a mobile phone Bluetooth App, the chip is programmed to be in the transmit mode with $0 \mathrm{dBm}$ output power. The top and bottom parts of the package are sealed by hot-melt adhesive to ensure protection in water environments. The active testing is displayed in Fig. 10(b). An open area is selected to avoid unwanted reflections and electromagnetic interference. The receiver is placed in water to measure its transmitted power while it is floating in water. A smartphone (with a custom Bluetooth App) is employed to measure the received power

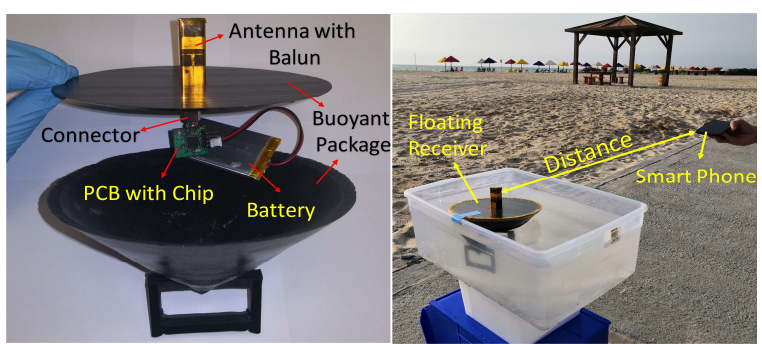

(a)

(b)

Fig. 10. Photos: (a) the proposed receiver system; (b) active tests.

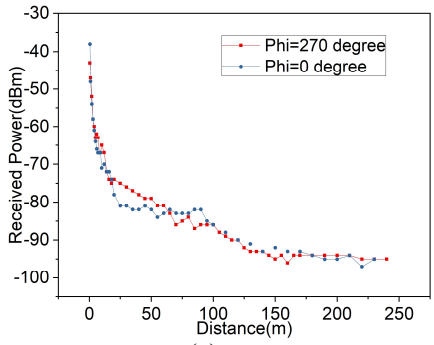

(a)

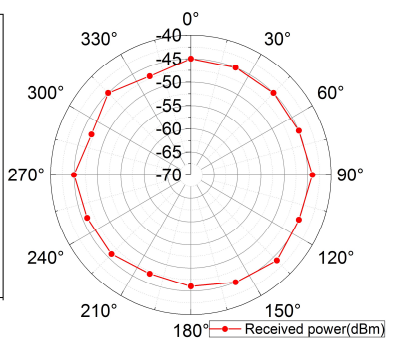

(b)
Fig. 11. Active test results: (a) range test; (b) azimuthal plane radiation pattern measured at a radius of $1 \mathrm{~m}$.

radiated from the floating antenna. The distance between the floating receiver and the smartphone is measured using a wheel stick.

The relationship between the received power and the distance at $\varphi=0^{\circ}$ and $\varphi=270^{\circ}$ is illustrated in Fig. 11(a). From Fig. 9 (b) we can observe that the maximum and the minimum gain values have been obtained at $\varphi=270^{\circ}$ (or 90 $0^{\circ}$ ) and $\varphi=0^{\circ}$ (or $-180^{\circ}$ )respectively, which means that the communication range at other angles should be between the values at $\varphi=0^{\circ}$ (or $180^{\circ}$ ) and $\varphi=270^{\circ}$ (or $90^{\circ}$ ) (between $240 \mathrm{~m}$ and $245 \mathrm{~m}$ ) theoretically, with a $-100 \mathrm{dBm}$ receiver sensitivity. The azimuthal radiation pattern recorded during the active testing is illustrated in Fig. 11(b), which is measured at a radius of $1 \mathrm{~m}$ in the azimuthal plane. The measured pattern is similar to the passive test results in Fig. 9(b), in which the direction of maximum received power is at $\pm y$-axis, further demonstrating that the buoyant package does not affect the antenna performance negatively.

\section{CONCLUSION}

An additively manufactured AiP with a quasi-isotropic radiation pattern is presented in this letter. This AiP is part of a marine animals monitoring system. The antenna is based on a folded SRR structure, which can generate simultaneously a pair of orthogonal electric and magnetic dipoles to achieve a quasiisotropic radiation pattern. The antenna is inexpensive due to low-cost 3D and inkjet printing utilized for the realization of the antenna. It is also compact in size due to the meandering of the antenna structure in addition to its implementation in the package of the driving electronics and battery. The measured bandwidth is $2.9 \%(70 \mathrm{MHz}$ at $2.4 \mathrm{GHz})$, and the measured maximum gain deviation in bandwidth is $3.6 \mathrm{~dB}$. The buoyant receiver can communicate within a range of $240 \mathrm{~m}$ in any direction. The results are promising for futuristic low-cost wireless sensor systems, particularly for marine applications. 


\section{REFERENCES}

[1] A.-Ladislao, "Environmental levels, toxicity and human exposure to tributyltin (TBT)-contaminated marine environment. A review, "in Environment International, vol. 34, no. 2, pp. 292-308, 2008.

[2] Favali, P. \& Co-Authors (2010). "Seafloor Observatory Science" in Proceedings of OceanObs'09: Sustained Ocean Observations and Information for Society (Vol. 2), Venice, Italy, 21-25 September 2009, Hall, J., Harrison, D.E. \& Stammer, D., Eds., ESA Publication WPP306, doi:10.5270/OceanObs09.cwp.28.

[3] B. Kuo, "SiP technology for wireless module miniaturization," in Proc. Additional Conf. Device Packag., HiTEC, HiTEN, CICMT, Jan. 2015, pp. 001956-001981.

[4] S. Ford and M. Despeisse, "Additive manufacturing and sustainability: An exploratory study of the advantages and challenges," J. Cleaner Prod., vol. 137, pp. 1573-1587, Nov. 2016.

[5] Z. Su, K. Klionovski, R. M. Bilal and A. Shamim, "3D Printed Antennaon-Package with Near-isotropic Radiation Pattern for IoT (WiFi Based) Applications," 2018 IEEE International Symposium on Antennas and Propagation \& USNC/URSI National Radio Science Meeting, Boston, MA, 2018, pp. 1431-1432.

[6] Z. Su, K. Klionovski, R. M. Bilal and A. Shamim, "A Dual Band Additively Manufactured 3-D Antenna on Package With Near-Isotropic Radiation Pattern," in IEEE Transactions on Antennas and Propagation, vol. 66, no. 7, pp. 3295-3305, July 2018.

[7] J. Kim and S. Nam, "A Compact Quasi-Isotropic Antenna Based on Folded Split-Ring Resonators," in IEEE Antennas and Wireless Propagation Letters, vol. 16, pp. 294-297, 2017.

[8] M. F. Farooqui, C. Claudel and A. Shamim, "An Inkjet-Printed Buoyant 3-D Lagrangian Sensor for Real-Time Flood Monitoring," in IEEE Transactions on Antennas and Propagation, vol. 62, no. 6, pp. 33543359, June 2014

[9] J. -. Kim and S. Nam, "Design of a compact dualband quasi-isotropic antenna," in Electronics Letters, vol. 53, no. 8, pp. 515-516, 1342017.

[10] Y. Pan and S. Zheng, "A Compact Quasi-Isotropic Shorted Patch Antenna," in IEEE Access, vol. 5, pp. 2771-2778, 2017.

[11] M. Tang and R. W. Ziolkowski, "A Study of Low-Profile, Broadside Radiation, Efficient, Electrically Small Antennas Based on Complementary Split Ring Resonators," in IEEE Transactions on Antennas and Propagation, vol. 61, no. 9, pp. 4419-4430, Sept. 2013.

[12] I. K. Kim and V. V. Varadan, "Electrically Small, Millimeter Wave Dual Band Meta-Resonator Antennas," in IEEE Transactions on Antennas and Propagation, vol. 58, no. 11, pp. 3458-3463, Nov. 2010.

[13] W. L. Stutzman and G. A. Thiele, Antenna Theory Design, 2nd ed. New York, NY, USA: Wiley, 1998.

[14] S. Kim, A. Shamim, A. Georgiadis, H. Aubert and M. M. Tentzeris, "Fabrication of Fully Inkjet-Printed Vias and SIW Structures on Thick Polymer Substrates," in IEEE Transactions on Components, Packaging and Manufacturing Technology, vol. 6, no. 3, pp. 486-496, March 2016. 